\title{
Rare Z-decay into light pseudoscalar bosons in the simplest little Higgs model
}

\author{
Lei Wang, Xiao-Fang Han \\ Department of Physics, Yantai University, Yantai 264005, China
}

\begin{abstract}
The simplest little Higgs model predicts a light pseudoscalar boson $\eta$ and opens up some new decay modes for $Z$-boson, such as $Z \rightarrow \bar{f} f \eta, Z \rightarrow \eta \eta \eta, Z \rightarrow \eta \gamma$ and $Z \rightarrow \eta g g$. We examine these decay modes in the parameter space allowed by current experiments, and find that the branching ratios can reach $10^{-7}$ for $Z \rightarrow \bar{b} b \eta, 10^{-8}$ for $Z \rightarrow \bar{\tau} \tau \eta$, and $10^{-8}$ for $Z \rightarrow \eta \gamma$, which should be accessible at the GigaZ option of the ILC. However, the branching ratios can reach $10^{-12}$ for $Z \rightarrow \eta \eta \eta$, and $10^{-11}$ for $Z \rightarrow \eta g g$, which are hardly accessible at the GigaZ option.
\end{abstract}

PACS numbers: 13.38.Dg,12.60.-i,14.80.Ec 


\section{INTRODUCTION}

Little Higgs theory [1] has been proposed as an interesting solution to the hierarchy problem. So far various realizations of the little Higgs symmetry structure have been proposed [2 5], which can be categorized generally into two classes [6]. One class use the product group, represented by the littlest Higgs model [3], in which the SM $S U(2)_{L}$ gauge group is from the diagonal breaking of two (or more) gauge groups. The other class use the simple group, represented by the simplest little Higgs model (SLHM) [4], in which a single larger gauge group is broken down to the $\mathrm{SM} S U(2)_{L}$.

Since these little Higgs models mainly alter the properties of the Higgs boson, hints of these models may be unraveled from various Higgs boson processes. The phenomenology of Higgs boson in these little Higgs models has been widely studied [7-10]. In addition to the SM-like Higgs boson $h$, the SLHM predicts a pseudoscalar boson $\eta$, whose mass can be as low as $\mathcal{O}(10 \mathrm{GeV})$. The constraint from the non-observation in the decay $\Upsilon \rightarrow \gamma \eta$ excludes $\eta$ with mass below 5-7 GeV [11]. Thus, the $Z$-decays into $\eta$ can be open for a light $\eta$, such as $Z \rightarrow \bar{f} f \eta(f=b, \tau), Z \rightarrow \eta \eta \eta, Z \rightarrow \eta \gamma$ and $Z \rightarrow \eta g g$.

The next generation $Z$ factory can be realized in the GigaZ option of the International Linear Collider (ILC)[12]. The ILC is a proposed electron-positron collider with tunable energy ranging from $400 \mathrm{GeV}$ to $500 \mathrm{GeV}$ and polarized beams in its first phase, and the GigaZ option corresponds to its operation on top of the resonance of $Z$-boson by adding a bypass to its main beam line. About $2 \times 10^{9} Z$ events can be generated in an operational year of $10^{7} \mathrm{~s}$ of GigaZ, which implies that the expected sensitivity to the branching ratio of $Z$-decay can be improved from $10^{-5}$ at the LEP to $10^{-8}$ at the GigaZ [12]. Therefore, it will offer an important opportunity to probe the new physics via the exotic or rare decays of $Z$-boson. The $Z$-boson flavor-changing neutral-current (FCNC) decays have been studied in many new physics models. For the lepton (quark) flavor violation decays, the branching ratios can be enhanced sizably compared with the SM predictions [13, 14]. In addition to the FCNC processes, the branching ratios for the processes of the $Z$-decay into light Higgs boson(s) in some new physics models can be accessible at the GigaZ option of the ILC [15 17]. In this paper, we will focus on the processes of the $Z$-decays into $\eta$ in the SLHM, namely $Z \rightarrow \bar{f} f \eta(f=b, \tau), Z \rightarrow \eta \eta \eta, Z \rightarrow \eta \gamma$ and $Z \rightarrow \eta g g$.

This work is organized as follows. In Sec. II we recapitulate the SLHM. In Sec. III we 
study the five decay modes, respectively. Finally, we give our conclusion in Sec. IV.

\section{SIMPLEST LITTLE HIGGS MODEL}

The SLHM is based on $\left[S U(3) \times U(1)_{X}\right]^{2}$ global symmetry. The gauge symmetry $S U(3) \times$ $U(1)_{X}$ is broken down to the SM electroweak gauge group by two copies of scalar fields $\Phi_{1}$ and $\Phi_{2}$, which are triplets under the $S U(3)$ with aligned VEVs $f_{1}$ and $f_{2}$. The uneaten five pseudo-Goldstone bosons can be parameterized as

$$
\Phi_{1}=e^{i t_{\beta} \Theta}\left(\begin{array}{c}
0 \\
0 \\
f_{1}
\end{array}\right), \quad \Phi_{2}=e^{-\frac{i}{t_{\beta}} \Theta}\left(\begin{array}{c}
0 \\
0 \\
f_{2}
\end{array}\right) \text {, }
$$

where

$$
\Theta=\frac{1}{f}\left[\left(\begin{array}{ccc}
0 & 0 & \\
0 & 0 & H \\
H^{\dagger} & 0
\end{array}\right)+\frac{\eta}{\sqrt{2}}\left(\begin{array}{lll}
1 & 0 & 0 \\
0 & 1 & 0 \\
0 & 0 & 1
\end{array}\right)\right],
$$

$f=\sqrt{f_{1}^{2}+f_{2}^{2}}$ and $t_{\beta} \equiv \tan \beta=f_{2} / f_{1}$. Under the $S U(2)_{L}$ SM gauge group, $\eta$ is a real scalar, while $H$ transforms as a doublet and can be identified as the SM Higgs doublet. The kinetic term in the non-linear sigma model is

$$
\mathcal{L}_{\Phi}=\sum_{j=1,2}\left|\left(\partial_{\mu}+i g A_{\mu}^{a} T^{a}-i \frac{g_{x}}{3} B_{\mu}^{x}\right) \Phi_{j}\right|^{2},
$$

where $g_{x}=g \tan \theta_{W} / \sqrt{1-\tan ^{2} \theta_{W} / 3}$ with $\theta_{W}$ being the electroweak mixing angle. As $\Phi_{1}$ and $\Phi_{2}$ develop their VEVs, the new heavy gauge bosons $Z^{\prime}, Y^{0}$, and $X^{ \pm}$get their masses proportional to $f$. A novel coupling of the SM-like Higgs boson $h$ can be derived from the Eq. (3),

$$
\mathcal{L}_{Z h \eta}=\frac{m_{Z}}{\sqrt{2} f}\left(t_{\beta}-\frac{1}{t_{\beta}}\right)\left(\eta \partial^{\mu} h-h \partial^{\mu} \eta\right) .
$$

The gauged $S U(3)$ symmetry promotes the SM fermion doublets into $S U(3)$ triplets. There are two possible gauge charge assignments for the fermions: the 'universal' embedding and the 'anomaly-free' embedding. The first choice is not favored by the electroweak precision data [4], so we focus on the second way of embedding. The quark Yukawa interactions for the third generation and the first two generations can be written respectively 
as

$$
\begin{gathered}
\mathcal{L}_{3}=i \lambda_{1}^{t} t_{1}^{c} \Phi_{1}^{\dagger} Q_{3}+i \lambda_{2}^{t} t_{2}^{c} \Phi_{2}^{\dagger} Q_{3}+i \frac{\lambda_{d}^{m}}{\Lambda} d_{m}^{c} \epsilon_{i j k} \Phi_{1}^{i} \Phi_{2}^{j} Q_{3}^{k}+h . c . \\
\mathcal{L}_{1,2}=i \lambda_{1}^{d_{n}} d_{1 n}^{c} Q_{n}^{T} \Phi_{1}+i \lambda_{2}^{d_{n}} d_{2 n}^{c} Q_{n}^{T} \Phi_{2}+i \frac{\lambda_{u}^{m n}}{\Lambda} u_{m}^{c} \epsilon_{i j k} \Phi_{1}^{* i} \Phi_{2}^{* j} Q_{n}^{k}+\text { h.c. }
\end{gathered}
$$

where $n=1,2$ are the first two generation indices; $i, j, k=1,2,3 ; Q_{3}=\left\{t_{L}, b_{L}, i T_{L}\right\}$ and $Q_{n}=\left\{d_{n L},-u_{n L}, i D_{n L}\right\} ; d_{m}^{c}$ runs over $\left(d^{c}, s^{c}, b^{c}, D^{c}, S^{c}\right) ; d_{1 n}^{c}$ and $d_{2 n}^{c}$ are linear combinations of $d^{c}$ and $D^{c}$ for $n=1$ and of $s^{c}$ and $S^{c}$ for $n=2 ; u_{m}^{c}$ runs over $\left(u^{c}, c^{c}, t^{c}, T^{c}\right)$. For simplicity, we assume the quark flavor mixing are small and neglect the mixing effects. Eqs. (5) and (6) contain the Higgs boson interactions and the mass terms for the three generations of quarks:

$$
\begin{aligned}
\mathcal{L}_{t} & \simeq-f \lambda_{2}^{t}\left[x_{\lambda}^{t} c_{\beta} t_{1}^{c}\left(-s_{1} t_{L}+c_{1} T_{L}\right) G_{1}(\eta)+s_{\beta} t_{2}^{c}\left(s_{2} t_{L}+c_{2} T_{L}\right) G_{2}(\eta)\right]+h . c . \\
\mathcal{L}_{d_{n}} & \simeq-f \lambda_{2}^{d_{n}}\left[x_{\lambda}^{d_{n}} c_{\beta} d_{1}^{c}\left(s_{1} d_{n L}+c_{1} D_{n L}\right) G_{1}^{*}(\eta)+s_{\beta} d_{2}^{c}\left(-s_{2} d_{n L}+c_{2} D_{n L}\right) G_{2}^{*}(\eta)\right]+h . c . \\
\mathcal{L}_{b} & \simeq-\frac{\lambda_{b}}{\Lambda} f^{2} s_{\beta} c_{\beta} s_{3} b^{c} b_{L} G_{3}(\eta)+h . c . \\
\mathcal{L}_{q} & \simeq-\frac{\lambda_{q}}{\Lambda} f^{2} s_{\beta} c_{\beta} s_{3} q^{c} q_{L} G_{3}^{*}(\eta)+h . c .(q=u, c)
\end{aligned}
$$

where

$$
\begin{aligned}
x_{\lambda}^{t} & \equiv \frac{\lambda_{1}^{t}}{\lambda_{2}^{t}}, \quad x_{\lambda}^{d_{n}} \equiv \frac{\lambda_{1}^{d_{n}}}{\lambda_{2}^{d_{n}}}, \quad s_{\beta} \equiv \frac{f_{2}}{\sqrt{f_{1}^{2}+f_{2}^{2}}}, \quad c_{\beta} \equiv \frac{f_{1}}{\sqrt{f_{1}^{2}+f_{2}^{2}}}, \\
s_{1} & \equiv \sin \frac{t_{\beta}(h+v)}{\sqrt{2} f}, \quad s_{2} \equiv \sin \frac{(h+v)}{\sqrt{2} t_{\beta} f}, \quad s_{3} \equiv \sin \frac{(h+v)\left(t_{\beta}^{2}+1\right)}{\sqrt{2} t_{\beta} f}, \\
G_{1}(\eta) & \equiv 1-i \frac{t_{\beta}}{\sqrt{2} f} \eta-\frac{t_{\beta}^{2}}{4 f^{2}} \eta^{2}, \quad G_{2}(\eta) \equiv 1+i \frac{1}{\sqrt{2} t_{\beta} f} \eta-\frac{1}{4 t_{\beta}^{2} f^{2}} \eta^{2} \\
G_{3}(\eta) & \equiv 1+i \frac{1}{\sqrt{2} f}\left(t_{\beta}-\frac{1}{t_{\beta}}\right) \eta-\frac{1}{4 f^{2}}\left(t_{\beta}-\frac{1}{t_{\beta}}\right)^{2} \eta^{2}
\end{aligned}
$$

with $h$ and $v$ being the SM-like Higgs boson field and its VEV, respectively. The mass eigenstates are obtained by mixing the corresponding interaction eigenstates, e.g., the mass eigenstates $\left(t_{m L}, T_{m L}\right)$ and $\left(t_{m}^{c}, T_{m}^{c}\right)$ are respectively the mixtures of $\left(t_{L}, T_{L}\right)$ and $\left(t^{c}, T^{c}\right)$. The diagonalization of the mass matrix in Eqs. (7) and (81) is performed numerically in our analysis, and the top quark $(T, D, d, S, s)$ couplings of $h$ and $\eta$ bosons can also be obtained without resort to any expansion of $v / f$. From Eqs. (9) and (10), we can get directly the couplings $-i \frac{m_{f}}{v} x_{f} h \bar{f} f$ and $\frac{m_{f}}{v} y_{f} \eta \bar{f} \gamma_{5} f(f=b, u, c)$ with

$$
x_{f}=\frac{v}{\sqrt{2} f}\left(t_{\beta}+\frac{1}{t_{\beta}}\right) \cot \frac{v}{\sqrt{2} f}\left(t_{\beta}+\frac{1}{t_{\beta}}\right), \quad y_{u}=y_{c}=-y_{b}=\frac{v}{\sqrt{2} f}\left(t_{\beta}-\frac{1}{t_{\beta}}\right) .
$$


The charged lepton couplings with $h$ and $\eta$ are the same as those of $b$ quark, but replacing $m_{b}$ with the corresponding lepton mass. Hereafter we denote the mass eigenstates without the subscript ' $m$ ' for simplicity.

The Yukawa and gauge interactions break the global symmetry and then provide a potential for the Higgs boson. However, the Coleman-Weinberg potential alone is not sufficient since the generated $h$ mass is too heavy and the new pseudoscalar $\eta$ is massless. Therefore, one can introduce a tree-level $\mu$ term which can partially cancel the $h$ mass [4, 9]:

$$
-\mu^{2}\left(\Phi_{1}^{\dagger} \Phi_{2}+\text { h.c. }\right)=-2 \mu^{2} f^{2} s_{\beta} c_{\beta} \cos \left(\frac{\eta}{\sqrt{2} s_{\beta} c_{\beta} f}\right) \cos \left(\frac{\sqrt{H^{\dagger} H}}{f c_{\beta} s_{\beta}}\right) .
$$

The Higgs potential becomes

$$
V=-m^{2} H^{\dagger} H+\lambda\left(H^{\dagger} H\right)^{2}-\frac{1}{2} m_{\eta}^{2} \eta^{2}+\lambda^{\prime} H^{\dagger} H \eta^{2}+\cdots
$$

where

$$
m^{2}=m_{0}^{2}-\frac{\mu^{2}}{s_{\beta} c_{\beta}}, \quad \lambda=\lambda_{0}-\frac{\mu^{2}}{12 s_{\beta}^{3} c_{\beta}^{3} f^{2}}, \quad \lambda^{\prime}=-\frac{\mu^{2}}{4 f^{2} s_{\beta}^{3} c_{\beta}^{3}}
$$

with $m_{0}$ and $\lambda_{0}$ being respectively the one-loop contributions to the $H$ mass and the quartic couplings from the contributions of fermion loops and gauge boson loops [4].

After EWSB, the coupling $h \eta \eta$ can be obtained from the term $\lambda^{\prime} H^{\dagger} H \eta^{2}$ in Eq. (14). The Higgs VEV and the masses of $h$ and $\eta$ are given by

$$
v^{2}=\frac{m^{2}}{\lambda}, \quad m_{h}^{2}=2 m^{2}, \quad m_{\eta}^{2}=\frac{\mu^{2}}{s_{\beta} c_{\beta}} \cos \left(\frac{v}{\sqrt{2} f s_{\beta} c_{\beta}}\right) .
$$

The Coleman-Weinberg potential involves the following parameters:

$$
f, x_{\lambda}^{t}, t_{\beta}, \mu, m_{\eta}, m_{h}, v
$$

Due to the modification of the observed $W$ gauge boson mass, $v$ is defined as [9]

$$
v \simeq v_{0}\left[1+\frac{v_{0}^{2}}{12 f^{2}} \frac{t_{\beta}^{4}-t_{\beta}^{2}+1}{t_{\beta}^{2}}-\frac{v_{0}^{4}}{180 f^{4}} \frac{t_{\beta}^{8}-t_{\beta}^{6}+t_{\beta}^{4}-t_{\beta}^{2}+1}{t_{\beta}^{4}}\right]
$$

where $v_{0}=246 \mathrm{GeV}$ is the SM Higgs VEV. Assuming that there are no large direct contributions to the potential from physics at the cutoff, we can determine other parameters in Eq. (17) from $f, t_{\beta}$ and $m_{\eta}$ with the definition of $v$ in Eq. (18). 


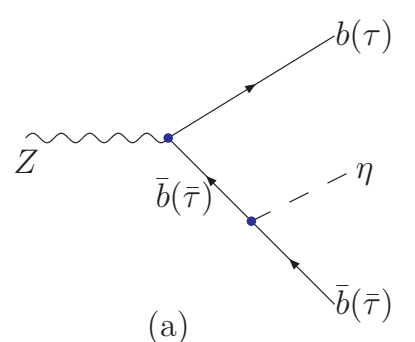

(a)

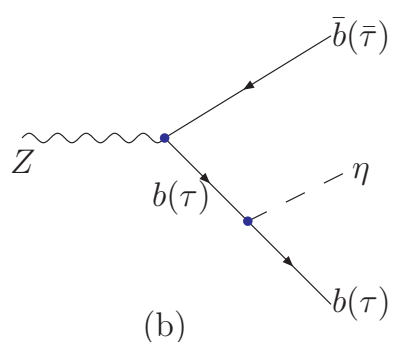

(b)

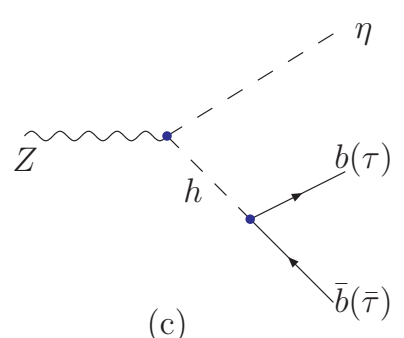

(c)

FIG. 1. Feynman diagrams for $Z \rightarrow \bar{f} f \eta(f=b, \tau)$ in the SLHM.

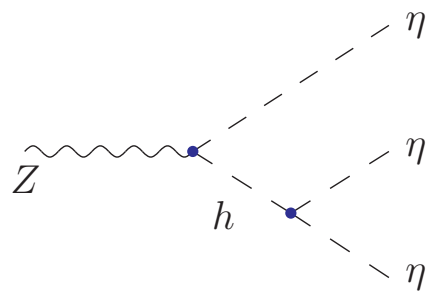

FIG. 2. Feynman diagrams for $Z \rightarrow \eta \eta \eta$ in the SLHM.

\section{THE RARE Z-DECAY INTO $\eta$}

In the SLHM, the rare $Z$-decays $Z \rightarrow \bar{f} f \eta(f=b, \tau), Z \rightarrow \eta \eta \eta, Z \rightarrow \eta \gamma$ and $Z \rightarrow \eta g g$ can be depicted by the Feynman diagrams shown in Fig. 1, Fig. 2, Fig. 3 and Fig. 4, respectively. For the decay $Z \rightarrow \eta \eta \eta$, ref. [15] shows the contributions of the scalar-loop diagrams can also be important for the large $h \eta \eta$ coupling. Here we do not consider the contributions of the loop diagram since the $h \eta \eta$ coupling does not have the large factor of enhancement in the SLHM (see $\lambda^{\prime}$ in Eq.(15)).

The calculations of the loop diagrams in Fig. 3 and Fig. 4 are straightforward. Each loop diagram is composed of some scalar loop functions [18] which are calculated by using LoopTools [19]. In appendix A, we list the amplitudes for $Z \rightarrow \bar{f} f \eta, Z \rightarrow \eta \eta \eta$ and $Z \rightarrow \eta \gamma$, respectively. The expressions for the amplitude of $Z \rightarrow \eta g g$ are very lengthy, which are not presented here.

In our calculations, we take $m_{t}=173.3 \mathrm{GeV}$ [20] and the other SM input parameters as ref. [21]. The free SLHM parameters are $f, t_{\beta}, m_{\eta}, x_{\lambda}^{d}$ and $x_{\lambda}^{s}$. As shown above, the parameters $x_{\lambda}^{t}, \mu, m_{h}$ can be determined by $f, t_{\beta}, m_{\eta}$ and $v$. To satisfy the bound of LEP2, we require that $m_{h}$ is larger than $114.4 \mathrm{GeV}[22]$. Certainly, due to the suppression of $h Z Z$ coupling [9], the LEP2 bound on $m_{h}$ should be loosened to some extent. The recent studies about $Z$ leptonic decay and $e^{+} e^{-} \rightarrow \tau^{+} \tau^{-} \gamma$ process at the $Z$-pole show that the scale $f$ 


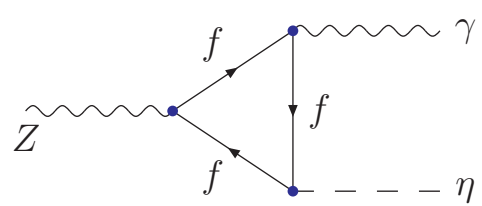

(a)

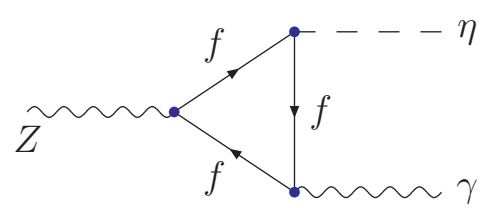

(b)

FIG. 3. Feynman diagrams for $Z \rightarrow \eta \gamma$ in the SLHM. $f$ denotes the charged fermions in SM, the new quarks $T, D$, and $S$.

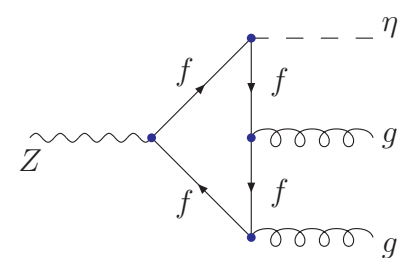

(a)

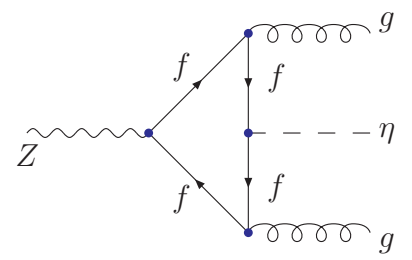

(c)

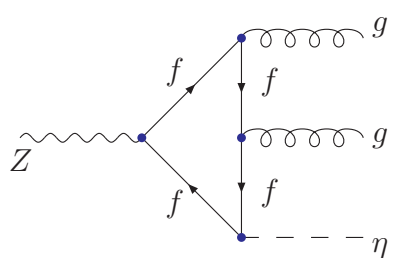

(b)

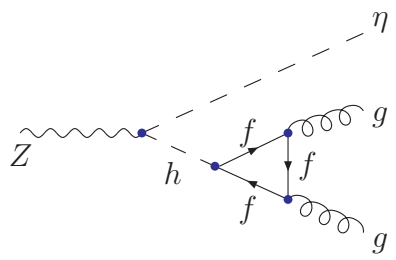

(d)

FIG. 4. Feynman diagrams for $Z \rightarrow \eta g g$ in the SLHM. $f$ denotes the SM quarks, the new quarks $T, D$, and $S$. The diagrams by exchanging the two gluons are not shown here.

should be respectively larger than 5.6 TeV and 5.4 TeV [23]. Here, we assume the new flavor mixing matrices in lepton and quark sectors are diagonal [6, 24, 25], so that $f$ and $t_{\beta}$ are free from the experimental constraints of the lepton and quark flavor violating processes. The large values of $f$ can suppress the SLHM predictions sizably. However, the factor $t_{\beta}$ in the couplings of $h$ and $\eta$ can be taken as a large value to cancel the suppression of $f$ partially. For the perturbation to be valid, $t_{\beta}$ cannot be too large for a fixed $f$. If we require $\mathcal{O}\left(v_{0}^{4} / f^{4}\right) / \mathcal{O}\left(v_{0}^{2} / f^{2}\right)<0.1$ in the expansion of $v, t_{\beta}$ should be below 28 for $f=5.6 \mathrm{TeV}$. In our calculations, we take $f=5.6 \mathrm{TeV}$ and $t_{\beta}=28,26,24$, respectively.

The small mass of the $d(s)$ quark requires one of the couplings $\lambda_{1}^{d}$ and $\lambda_{2}^{d}\left(\lambda_{1}^{s}\right.$ and $\left.\lambda_{2}^{s}\right)$ to be very small, so there is almost no mixing between the SM down-type quarks and their heavy partners. We assume $\lambda_{1}^{d}\left(\lambda_{1}^{s}\right)$ is small, and take $x_{\lambda}^{d}=1.1 \times 10^{-4}\left(x_{\lambda}^{s}=2.1 \times 10^{-3}\right)$, which can make the masses of $D$ and $S$ be in the range of $1 \mathrm{TeV}$ and $2 \mathrm{TeV}$ for other parameters taken in our calculations. In fact, our results show that different choices of $x_{\lambda}^{d}$ and $x_{\lambda}^{s}$ can not have sizable effects on the result. 

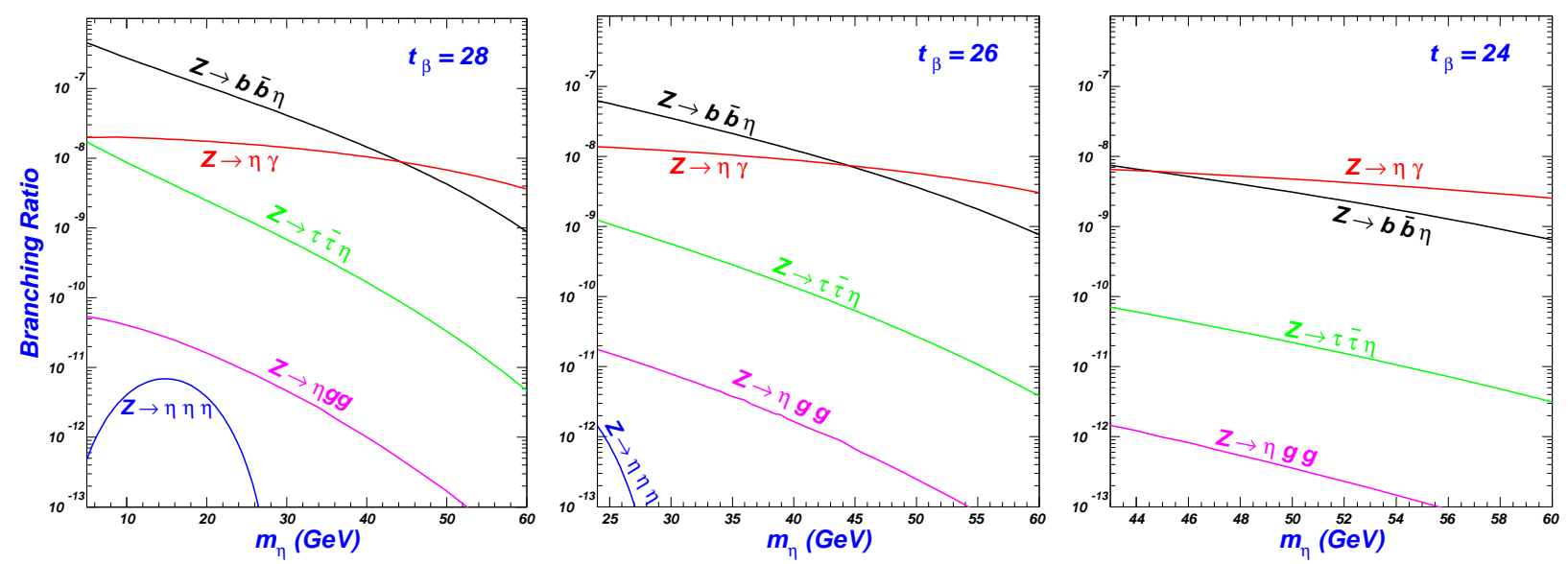

FIG. 5. For $f=5.6 \mathrm{TeV}$, the branching ratios of $Z \rightarrow \bar{f} f \eta(f=b, \tau), Z \rightarrow \eta \eta \eta, Z \rightarrow \eta \gamma$ and $Z \rightarrow \eta g g$ versus the $\eta$ boson mass. The incomplete lines for $t_{\beta}=26$ and 24 show the lower bounds of the $\eta$ mass, respectively.

In Fig. 5, we plot the decay branching ratios of $Z \rightarrow \bar{f} f \eta(f=b, \tau), Z \rightarrow \eta \eta \eta, Z \rightarrow \eta \gamma$ and $Z \rightarrow \eta g g$ versus the $\eta$ mass for $t_{\beta}=28,26,24$, respectively. Because of the constraint of $m_{h}>114.4 \mathrm{GeV}$, the lower bound of $m_{\eta}$ is enhanced for the small $t_{\beta}$. Fig. 5 shows that the ratios can reach $7 \times 10^{-12}$ for $Z \rightarrow \eta \eta \eta$ with $m_{\eta}=15 \mathrm{GeV}$ and $t_{\beta}=28$, and $5 \times 10^{-11}$ for $Z \rightarrow \eta g g$ with $m_{\eta}=5 \mathrm{GeV}$ and $t_{\beta}=28$, which are too small to be detectable at the Gigaz option of the ILC. The reason should be partly from the suppression of three-body phase space. Besides, for the decay $Z \rightarrow \eta g g$, we find there is a strong cancellation between the contributions of different diagrams shown in Fig. 4, which can reduce the branching ratio sizably. As the increasing of $m_{\eta}$, the phase space of $Z \rightarrow \eta \eta \eta$ is suppressed, and the $h \eta \eta$ coupling which is proportional to $m_{\eta}^{2}$ is enhanced, so the ratio reaches its peak for $m_{\eta}=15$ $\mathrm{GeV}$ and $t_{\beta}=28$. In addition to the couplings $Z \eta h$ and $h \eta \eta$ which are suppressed by the scale $f$, the factor $\frac{1}{\hat{s}-m_{h}^{2}}$ contributed by the intermediate state $h$ shown in Fig. 2 (see Eq. (A4)) can suppress the branching ratio of $Z \rightarrow \eta \eta \eta$ sizably.

Fig. 5 shows that the ratios can reach $10^{-7}$ for $Z \rightarrow \eta \bar{b} b, 10^{-8}$ for $Z \rightarrow \eta \bar{\tau} \tau$, and $10^{-8}$ for $Z \rightarrow \eta \gamma$ for a light $\eta$ boson with $t_{\beta}=28$, which should be accessible at the Gigaz option. The three ratios decrease with increasing of $m_{\eta}$, especially for the decays $Z \rightarrow \eta \bar{b} b$ and $Z \rightarrow \eta \bar{\tau} \tau$. For $t_{\beta}=26$, the lower bound of $m_{\eta}$ is enhanced to $24 \mathrm{GeV}$ to satisfy the constraint of $m_{h}>114.4 \mathrm{GeV}$, and the ratios of $Z \rightarrow \eta \bar{\tau} \tau$ is below $10^{-8}$, which is hardly accessible at the Gigaz option. For $t_{\beta}=24$, the lower bound of $m_{\eta}$ is enhanced to $43 \mathrm{GeV}$, and the ratios of the three decays are too small to detectable at the Gigaz option. The 
branching ratio of $Z \rightarrow \eta \bar{b} b$ always dominates over $Z \rightarrow \eta \bar{\tau} \tau$ since the coupling $\eta \bar{b} b$ is larger than $\eta \bar{\tau} \tau$, and the former can be enhanced by the color factor. Although the decay $Z \rightarrow \eta \gamma$ occurs at one-loop level, the branching ratio can be comparable with those of $Z \rightarrow \eta \bar{f} f$ because of the large couplings $\eta \bar{t} t$ and $\eta \bar{T} T$, and suppression of the three-body phase space for $Z \rightarrow \eta \bar{f} f$.

\section{CONCLUSION}

In the framework of the simplest little Higgs model, we studied the rare Z-decays $Z \rightarrow$ $\bar{f} f \eta, Z \rightarrow \eta \eta \eta, Z \rightarrow \eta \gamma$ and $Z \rightarrow \eta g g$. In the parameter space allowed by current experiments, the branching ratios can reach $10^{-7}$ for $Z \rightarrow \bar{b} b \eta, 10^{-8}$ for $Z \rightarrow \bar{\tau} \tau \eta$, and $10^{-8}$ for $Z \rightarrow \eta \gamma$, which should be accessible at the GigaZ option of the ILC. However, the branching ratios can reach $10^{-12}$ for $Z \rightarrow \eta \eta \eta$, and $10^{-11}$ for $Z \rightarrow \eta g g$, which are too small to be detectable at the Gigaz.

\section{ACKNOWLEDGMENT}

We thank Junjie Cao, Zhaoxia Heng, Wenlong Sang, and Jin Min Yang for discussions. This work was supported in part by the National Natural Science Foundation of China (NNSFC) under grant No. 11005089, and by the Foundation of Yantai University under Grant No. WL10B24.

\section{Appendix A: Amplitudes of $Z \rightarrow \bar{f} f \eta, Z \rightarrow \eta \eta \eta$ and $Z \rightarrow \eta \gamma$}

Here we give the amplitude of the process $Z\left(p_{1}, \epsilon_{1}\right) \rightarrow \bar{f}\left(p_{4}\right) f\left(p_{3}\right) \eta\left(p_{2}\right)(f=b, \tau)$. The expressions are given by

$$
M=M_{a}+M_{b}+M_{c}
$$

where

$$
\begin{aligned}
& M_{a}=-\frac{1}{\left(p_{1}-p_{4}\right)^{2}-m_{f}^{2}} \bar{u}\left(p_{3}\right)\left(g_{L}^{\eta} P_{L}+g_{R}^{\eta} P_{R}\right)\left(\not p_{1}-\not p_{4}+m_{f}\right) \gamma^{\mu}\left(g_{L}^{Z} P_{L}+g_{R}^{Z} P_{R}\right) v\left(p_{4}\right) \epsilon_{1}\left(p_{1}\right), \\
& M_{b}=-\frac{1}{\left(p_{1}-p_{3}\right)^{2}-m_{f}^{2}} \bar{u}\left(p_{3}\right) \gamma^{\mu}\left(g_{L}^{Z} P_{L}+g_{R}^{Z} P_{R}\right)\left(\not p_{3}-\not p_{1}+m_{f}\right)\left(g_{L}^{\eta} P_{L}+g_{R}^{\eta} P_{R}\right) v\left(p_{4}\right) \epsilon_{1}\left(p_{1}\right), \\
& M_{c}=\frac{g_{h f \bar{f}} g_{Z h \eta}}{\left(p_{3}+p_{4}\right)^{2}-m_{h}^{2}} \bar{u}\left(p_{3}\right) v\left(p_{4}\right)\left(p_{1}-2 p_{3}-2 p_{4}\right)^{\mu} \epsilon_{\mu}\left(p_{1}\right) .
\end{aligned}
$$


The amplitude of $Z\left(p_{1}, \epsilon_{1}\right) \rightarrow \eta\left(p_{2}\right) \eta\left(p_{3}\right) \eta\left(p_{4}\right)$ is as follows:

$$
M=M_{1}\left(p_{1}, p_{2}, p_{3}, p_{4}\right)+M_{1}\left(p_{1}, p_{3}, p_{2}, p_{4}\right)+M_{1}\left(p_{1}, p_{4}, p_{3}, p_{2}\right),
$$

where

$$
M_{1}\left(p_{1}, p_{2}, p_{3}, p_{4}\right)=\frac{g_{h \eta \eta} g_{Z h \eta}}{\hat{s}-m_{h}^{2}}\left(p_{3}+p_{4}-p_{2}\right)^{\mu} \epsilon_{\mu}\left(p_{1}\right) \text { with } \hat{s}=\left(p_{3}+p_{4}\right)^{2} .
$$

The expressions for the amplitude of $Z\left(p_{1}, \epsilon_{1}\right) \rightarrow \eta\left(p_{3}\right) \gamma\left(p_{2}, \epsilon_{2}\right)$ can be given by

$$
\begin{aligned}
M= & \frac{N_{c f} Q_{f}}{4 \pi^{2}}(A+B)\left[\epsilon_{1} \cdot \epsilon_{2}^{*}\left(-2 m_{f} C_{\nu} p_{2}^{\nu}+\frac{m_{f}}{2}-m_{f} C_{\mu \nu} g^{\mu \nu}+m_{f} p_{2} \cdot p_{3} C_{0}+m_{f}^{3} C_{0}\right)\right. \\
& +p_{2} \cdot \epsilon_{2}^{*}\left(2 m_{f} C_{\mu} \epsilon_{1}^{\mu}-m_{f} p_{3} \cdot \epsilon_{1} C_{0}\right)+p_{2} \cdot \epsilon_{1}\left(2 m_{f} C_{\nu} \epsilon_{2}^{* \nu}-m_{f} p_{3} \cdot \epsilon_{2}^{*} C_{0}\right) \\
& \left.-2 m_{f} p_{3} \cdot \epsilon_{1} C_{\nu} \epsilon_{2}^{* \nu}+4 m_{f} C_{\mu \nu} \epsilon_{1}^{\mu} \epsilon_{2}^{* \nu}\right]+i \frac{N_{c f} Q_{f}}{4 \pi^{2}}(C-D) m_{f} \varepsilon_{\nu \alpha \mu \beta} \epsilon_{1}^{\mu} \epsilon_{2}^{* \nu} p_{2}^{\alpha} p_{3}^{\beta} C_{0},
\end{aligned}
$$

where $N_{c f}$ and $Q_{f}$ denote the color factor and the electric charge for the fermion $f$, respectively; $A=\frac{1}{2}\left(g_{L}^{Z} g_{R}^{\eta}+g_{R}^{Z} g_{L}^{\eta}\right), B=\frac{1}{2}\left(g_{L}^{Z} g_{L}^{\eta}+g_{R}^{Z} g_{R}^{\eta}\right), C=\frac{1}{2}\left(g_{L}^{Z} g_{R}^{\eta}-g_{R}^{Z} g_{L}^{\eta}\right)$, and $D=\frac{1}{2}\left(g_{L}^{Z} g_{L}^{\eta}-\right.$ $\left.g_{R}^{Z} g_{R}^{\eta}\right)$. The parameters $g_{L}^{Z}, g_{R}^{Z}, g_{L}^{\eta}$ and $g_{R}^{\eta}$ are respectively from the couplings $i \bar{f} \gamma^{\mu}\left(g_{L}^{Z} P_{L}+\right.$ $\left.g_{R}^{Z} P_{R}\right) f Z$ and $\bar{f}\left(g_{L}^{\eta} P_{L}+g_{R}^{\eta} P_{R}\right) f \eta$ with $P_{L, R}=\frac{1 \mp \gamma^{5}}{2} . C_{0}, C_{\mu}$ and $C_{\mu \nu}$ are respectively the 3point loop functions, and their dependence is given by $C_{0, \mu, \mu \nu} \equiv C_{0, \mu, \mu \nu}\left(p_{2},-p_{1}, m_{f}, m_{f}, m_{f}\right)$.

[1] N. Arkani-Hamed, A. G. Cohen and H. Georgi, Phys. Lett. B 513, 232 (2001); N. ArkaniHamed, A. G. Cohen, E. Katz, A. E. Nelson, T. Gregoire and J. G. Wacker, JHEP 0208, 021 (2002).

[2] D. E. Kaplan and M. Schmaltz, JHEP 0310, 039 (2003); I. Low, W. Skiba, and D. Smith, Phys. Rev. D 66, 072001 (2002); S. Chang and J. G. Wacker, Phys. Rev. D 69, 035002 (2004); T. Gregoire, D. R. Smith, and J. G. Wacker, Phys. Rev. D 69, 115008 (2004); W. Skiba and J. Terning, Phys. Rev. D 68, 075001 (2003); S. Chang, JHEP 0312, 057 (2003); H. Cai, H.-C. Cheng, and J. Terning, JHEP 0905, 045 (2009); A. Freitas, P. Schwaller, and D. Wyler, JHEP 0912, 027 (2009).

[3] N. Arkani-Hamed, A. G. Cohen, E. Katz and A. E. Nelson, JHEP 0207, 034 (2002).

[4] M. Schmaltz, JHEP 0408, 056 (2004).

[5] H. C. Cheng, I. Low, JHEP 0309, 051 (2003); JHEP 0408, 061 (2004); H. C. Cheng, I. Low and L. T. Wang, Phys. Rev. D 74, 055001 (2006); J. Hubisz and P. Meade, Phys. Rev. D 71, $035016(2005)$. 
[6] T. Han, H. E. Logan and L. T. Wang, JHEP 0601, 099 (2006).

[7] T. Han, H. E. Logan, B. McElrath, L. T. Wang, Phys. Lett. B 563, 191 (2003) [Erratum-ibid. B 603, 257 (2004)].

[8] C. R. Chen, K. Tobe, C. P. Yuan, Phys. Lett. B 640, 263 (2006); K. Hsieh, C. P. Yuan, Phys. Rev. D 78,053006 (2008); G. A. Gonzalez-Sprinberg etal., Phys. Rev. D 71, 035003 (2005); L. Wang, et al., Phys. Rev. D 75, 074006 (2007); Phys. Rev. D 76, 017702 (2007); Phys. Rev. D 77, 015020 (2008); Phys. Rev. D 79, 055013 (2009); Phys. Rev. D 82, 095009 (2010); R. S. Hundi etal., Phys. Lett. B 649, 280 (2007); P. Kai, et al., Phys. Rev. D 76, 015012 (2007); C. X. Yue, N. Zhang, Europhys. Lett. 77, 51003 (2007); S. Yang, Phys. Lett. B 675, 352-355 (2009); L. Wang, F. Xu, J. M. Yang, JHEP 1001, 107 (2010); X. F. Han, L. Wang, J. M. Yang, Nucl. Phys. B 825, 222 (2010); E. Asakawa etal., Phys. Rev. D 82, 115002 (2010); C. Dib, R. Rosenfeld, A. Zerwekh, JHEP 0605, 074 (2006); J. J. Liu, et al., Phys. Rev. D 70, 015001 (2004); K. Cheung etal., Phys. Rev. D 78, 055015 (2008).

[9] K. Cheung and J. Song, Phys. Rev. D 76, 035007 (2007).

[10] W. Kilian, D. Rainwater and J. Reuter, Phys. Rev. D 71, 015008 (2005).

[11] N. Brambilla, et al. (Quarkonium Working Group), arXiv:hep-ph/0412158; W. M. Yao, et al. (Particle Data Group), J. Phys. G 33, 1 (2006); R. Balest, et al. (CLEO Collaboration), Phys. Rev. D 51, 2053 (1995).

[12] J. A. Aguilar-Saavedra et al., hep-ph/0106315.

[13] J. I. Illana, M. Masip, Phys. Rev. D 67, 035004 (2003); M. A. Mughal et al., Phys. Lett. B 417, 87 (1998); J. Cao, Z. Xiong, J. M. Yang, Eur. Phys. Jour. C 32, 245 (2004); J. Cao, L. Wu, J. M. Yang, Nucl. Phys. B 829, 370 (2010); D. Delepine and F. Vissani, Phys. Lett. B 522, 95 (2001); M. Frank, Phys. Rev. D 65, 033011 (2002); A. Ghosal, Y. Koide, and H. Fusaoka, Phys. Rev. D 64, 053012 (2001); P. Langaker and M. Plumacher, Phys. Rev. D 62, 013006 (2000); C. Yue et al., Phys. Lett. B 536, 67 (2002); E. O. Iltan and I. Turan, Phys. Rev. D 65, 013001 (2002); A. Ilakovac and A. Pilaftis, Nucl. Phys. B 437, 491 (1995); C.-X. Yue etal., Phys. Rev. D 78, 095006 (2008).

[14] B. Grzadkowski et al., Phys. Lett. B 268, 106 (1991); M. Chemtob and G. Moreau, Phys. Rev. D 59, 116012 (1999); D. Atwood et al., Phys. Rev. D 66, 093005 (2002); G. T. Park, and T. K. Kuo, Phys. Rev. D 42, 3879 (1990); X. Zhang and B. L. Young, Phys. Rev. D 51, 6584 (1995); J. Roldanet al., Phys. Lett. B 283, 389 (1992); X. L. Wang, G. R. Lu, and Z. J. 
Xiao, Phys. Rev. D 51, 4992 (1995); X.-F. Han etal., Phys. Rev. D 78, 075017 (2008); J. M. Yang, arXiv:1006.2594.

[15] J. Cao, Z. Heng, J. M. Yang, JHEP 1011, 110 (2010).

[16] D. Chang and W. Y. Keung, Phys. Rev. Lett. 77, 3732 (1996).

[17] E. Keith and E. Ma, Phys. Rev. D 57, 2017 (1998); F. Larios, G. Tavares-Velasco and C. P. Yuan, Phys. Rev. D 64, 055004 (2001); Phys. Rev. D 66, 075006 (2002).

[18] G. 't Hooft and M. J. G. Veltman, Nucl. Phys. B 153, 365 (1979).

[19] T. Hahn and M. Perez-Victoria, Comput. Phys. Commun. 118, 153 (1999); T. Hahn, Nucl. Phys. Proc. Suppl. 135, 333 (2004).

[20] Tevatron Electroweak Working Group, CDF, D0 Collaborations, arXiv:1007.3178.

[21] C. Amsler, et al., Phys. Lett. B 667, 1 (2008).

[22] R. Barate, et al. (LEP Working Group for Higgs boson searches), Phys. Lett. B 565, 61 (2003).

[23] A. G. Dias, C. A. de S. Pires, P. S. Rodrigues da Silva, Phys. Rev. D 77, 055001 (2008); A. Gutierrez-Rodriguez, Mod. Phys. Lett. A 25, 703-713 (2010).

[24] J. I. Illana, M. D. Jenkins, Acta Phys. Polon. B 40, 3143 (2009).

[25] F. d. Aguila, J. I. Illana, M. D. Jenkins, Nucl. Phys. Proc. Suppl. 205-206, 158-163 (2010). 